\title{
Resistência à Mancha Preta dos Citros Avaliada por Curvas de Progresso da Doença*
}

\author{
Marcel B. Spósito**1, Renato B. Bassanezi ${ }^{1}$ \& Lilian Amorim**2 \\ ${ }^{1}$ Departamento Científico, Fundecitrus, Cx. Postal 391, CEP 14801-970, Araraquara, SP, e-mail: marcel@fundecitrus.com.br; \\ ${ }^{2}$ Departamento de Entomologia, Fitopatologia e Zoologia Agrícola, ESALQ-USP, Cx. Postal 9, CEP 13418-900, Piracicaba, \\ SP, e-mail: liamorim@esalq.usp.br
}

(Aceito para publicação em 17/06/2004)

Autor para correspondência: Marcel B. Spósito

SPÓSITO, M.B., BASSANEZI, R.B. \& AMORIM, L. Resistência à mancha preta dos citros avaliada por curvas de progresso da doença. Fitopatologia Brasileira 29:532-537. 2004.

\section{RESUMO}

A mancha preta dos citros (Citrus spp.), causada por Guignardia citricarpa, vem causando sérios prejuízos à citricultura paulista. O parque citrícola está alicerçado em praticamente quatro variedades de copa de laranja doce (Citrus sinensis): 'Hamlin’, 'Pera', 'Valência’ e 'Natal'. A literatura cita as variedades tardias ('Valência' e 'Natal') como as mais suscetíveis, em razão da elevada severidade da doença nos frutos dessas variedades por ocasião da colheita, mas não há informações sobre o progresso temporal da doença no campo. A resistência das variedades 'Hamlin' (precoce), 'Pera' (meia estação) e 'Valência' (tardia) à mancha preta dos citros foi avaliada em pomar comercial, sob infecção natural. Avaliaram-se a severidade e a incidência da doença em 100 frutos de 100 plantas de cada variedade, a cada 15 dias, desde a primeira observação dos sintomas no campo até o momento da colheita. O modelo monomolecular foi ajustado às curvas de progresso da incidência e da severidade da doença para as três variedades. As três variedades apresentaram a mesma taxa de progresso da doença $(r)$. Concluiu-se que as variedades 'Hamlin', 'Pera' e 'Valência' possuem o mesmo nível de suscetibilidade à mancha preta dos citros.

Palavras-chave adicionais: Citrus sinensis, Phyllosticta citricarpa, epidemiologia, análise temporal.

\section{ABSTRACT}

Resistance to citrus black spot by the analyses of disease progress curves

Citrus (Citrus spp.) black spot, caused by Guignardia citricarpa, is responsible for serious damage to citriculture in the State of São Paulo. The main varieties of oranges (Citrus sinensis) in São Paulo are 'Hamlin', 'Pera', 'Valência' and 'Natal'. The literature suggests that late varieties such as 'Valência' and 'Natal' are most susceptible to citrus black spot, because these varieties are severely affected at harvest time. Nevertheless, there is no information about the temporal disease progress in the field. Resistance of the orange

\section{INTRODUÇÃO}

A mancha preta dos citros (Citrus spp.) (MPC), cujo agente causal é o fungo Guignardia citricarpa Kiely, foi relatada pela primeira vez em pomares comerciais no Estado de São Paulo em 1992, na região de Limeira (Goes \& Feichtenberger, 1993). Os sintomas ocasionados pela doença depreciam os frutos para a comercialização in natura no mercado interno, assim como restringem as exportações (Aguilar-Vildoso et al., 2002). A MPC também causa a queda prematura dos frutos (Timmer, 1999), podendo levar a reduções de até 80\% na produção (Klotz, 1978). Contudo, os sintomas

\footnotetext{
*Parte da tese de doutorado do primeiro autor na Escola Superior de Agricultura "Luiz de Queiroz", Universidade de São Paulo (2003), parcialmente financiada pela FAPESP (01/05508-5).

**Bolsistas do CNPq.
}

varieties ‘Hamlin' (early), ‘Pera' (mid-season), and 'Valência’ (late) to citrus black spot was assessed in a commercial grove, under conditions of natural infection. Disease severity and disease incidence were assessed in 100 fruits of 100 plants of each variety, every 15 days, from the first appearance of symptoms in the field until harvest. The monomolecular model was fitted to the data of the disease progress curves for the three varieties. The rate parameter $(r)$ of the disease progress curve was not different for the three varieties. Varieties 'Hamlin', 'Pera' and 'Valência' present the same degree of susceptibility to the MPC. da MPC ficam restritos ao flavedo dos frutos (Cardoso Filho, 2003), não havendo interferência em sua qualidade interna. Frutos doentes podem, portanto, ser utilizados no processamento para produção de suco (Aguilar-Vildoso et al., 2002).

A indústria paulista de suco de laranja está alicerçada num parque citrícola de aproximadamente 197 milhões de plantas, 98,2\% das quais são de apenas quatro variedades de laranjeiras: a precoce 'Hamlin' (1,7\%), a de meia estação 'Pera' (48,5\%) e as tardias 'Valência' (27,5\%) e 'Natal' (20,5\%) (FNP Consultoria \& Comércio, 2002). A MPC afeta todas as variedades de laranjeiras doces (Alcoba et al., 2000; AguilarVildoso et al., 2002) e a expressão de seus sintomas está relacionada com a época de maturação das variedades, sendo tanto maior quanto mais madura estiver a fruta (Feichtenberger, 1996). O período de latência varia entre quatro e seis meses 
(Timmer, 1999). Altas temperaturas e intensa radiação solar são condições ambientais que favorecem a expressão dos sintomas (Kotzé, 1963). Com isto, alguns autores atribuem a maior intensidade da doença em variedades tardias, ao maior período de exposição de seus frutos a condições favoráveis para a expressão dos sintomas (Feichtenberger, 1996). Entretanto, outros autores atribuem a maior expressão de sintomas de mancha preta em variedades tardias à sua maior suscetibilidade (Kiely, 1948; Goes, 1998; Timmer, 1999).

A epidemiologia comparativa tem como objetivo identificar similaridades ou diferenças entre epidemias, baseada na natureza ou no comportamento da curva de progresso da doença (Campbell \& Madden, 1990). A comparação de curvas de progresso de doenças em variedades com diferentes níveis de resistência tem sido um dos objetos de estudo da epidemiologia comparativa. $\mathrm{O}$ ajuste de modelos e a comparação das áreas abaixo da curva de progresso da doença (Keinath \& Farnham, 1997; Silva et al., 1998) são métodos usualmente aplicados com esse objetivo. A curva de progresso da doença integra os efeitos do patógeno, do hospedeiro e do ambiente em um único gráfico (Campbell \& Madden, 1990). Pode-se, portanto, comparar a resistência de variedades por meio da taxa de progresso da doença, desde que as plantas sejam submetidas a condições similares de clima e sob a mesma população patogênica.

Este trabalho teve como objetivo comparar a suscetibilidade dos frutos de três variedades de laranjeiras doces [Citrus sinensis (L.) Osbeck] com diferentes períodos de maturação, 'Hamlin’ (precoce), 'Pera' (meia estação) e 'Valência’ (tardia) à mancha preta dos citros, em condições naturais de infecção.

\section{MATERIALEMÉTODOS}

As curvas de progresso analisadas neste estudo foram obtidas em experimento conduzido em pomar comercial no município paulista de Mogi Guaçu, com alta pressão de inóculo de mancha preta dos citros, onde foram selecionados três talhões de laranjeiras doces : um talhão da variedade precoce 'Hamlin', com nove anos de idade e espaçamento entre plantas de 8 x 4 m; um talhão da variedade de meia estação 'Pera', com nove anos de idade e espaçamento entre plantas de 7 x 3,5 m; e um talhão da variedade tardia 'Valência', com oito anos de idade e espaçamento entre plantas de 7,5 x 3,5 m. Em cada talhão foi marcado um bloco de 100 plantas (cinco ruas de 20 plantas) onde avaliou-se, a partir da primeira constatação dos sintomas da doença (13 de março de 2001), a incidência (porcentagem de frutos sintomáticos) e a severidade (porcentagem de casca dos frutos afetada pela doença) em 100 frutos por planta, a cada 15 dias, até a sua colheita (18 de julho de 2001 para a variedade ‘Hamlin’ e 31 agosto de 2001 para as variedades 'Pera' e 'Valência'). Para avaliar a severidade da doença, utilizou-se escala diagramática contendo os principais sintomas observados no campo: a "mancha dura" e a "falsa melanose” (Spósito et al., 2003). Os modelos matemáticos mais usados em estudos epidemiológicos: monomolecular
$\left(Y=b_{1}{ }^{*}\left(1-b_{2}{ }^{*} \exp (-r t)\right)\right)$, logístico $\left(Y=b_{1} /\left(1+b_{2}{ }^{*} \exp (-r t)\right)\right)$ e Gompertz $\left(Y=b_{1}^{*} \exp \left(-b_{2} \exp (-r t)\right)\right)$, em que $Y$ é a incidência ou severidade da doença, $b_{1}$ representa a assíntota máxima estimada pelo modelo, $b_{2}$ é a constante de integração igual a 1$\mathrm{y}_{0}\left(\mathrm{y}_{0}=\right.$ inóculo inicial), $r$ representa a taxa de progresso da doença e $t$, o tempo, foram ajustados às curvas de progresso da doença (Bergamin Filho \& Amorim, 1996), por meio de regressões não lineares, com o programa Statistica 6.0 (Tulsa, Statsoft). A escolha do melhor modelo foi feita pelo maior valor do coeficiente de determinação $\left(R^{2}\right)$ da regressão não-linear entre doença e tempo e pela ausência de padrão dos resíduos (Cornell \& Berger, 1987; Neter et al., 1996). As taxas de progresso da doença $(r)$ das curvas de incidência e de severidade da MPC obtidas com o modelo de melhor ajuste foram comparadas pelo teste $t$. Comparou-se também pelo teste $t$ a assíntota máxima estimada $\left(b_{1}\right)$ e a constante de integração $\left(b_{2}\right)$ das três variedades, relacionando-as ao máximo de doença observada e ao início da expressão dos sintomas.

Foram determinados, também, em cada período de avaliação, o diâmetro médio e a cor da casca de 50 frutos da área de cada variedade. O cálculo da cor da casca foi feito através de método objetivo, utilizando para isto colorímetro Minolta, onde se mediu o índice de cor (IC) pela fórmula: IC=1000 × $a / L \times b$, em que $L$ é a luminosidade, $a$ é a variação entre a cor verde e vermelha e o $b$ a variação entre a cor azul e a amarela (Mazzuz, 1996). Este índice varia de-20 a +20; quanto mais negativo, mais verde e quanto mais positivo, mais alaranjado está o fruto. O valor zero representa a cor amarela.

\section{RESULTADOS E DISCUSSÃO}

Um dos aspectos mais importantes na análise temporal de epidemias é a seleção de um modelo apropriado para descrever a curva de progresso da doença. Esta seleção tem como objetivo estimar parâmetros que são utilizados na análise estatística para a comparação entre curvas de progresso de doenças (Campbell \& Madden, 1990).

Os modelos matemáticos testados no ajuste das curvas de progresso da mancha preta dos citros (monomolecular, logístico e Gompertz) mostraram coeficientes de determinação $\left(R^{2}\right)$ muito similares para as três variedades avaliadas, 'Hamlin', 'Pera’e 'Valência' (Tabela 1). Não foram observados padrões nos resíduos dos modelos ajustados aos dados de severidade para quaisquer variedades, tampouco para os dados de incidência das variedades 'Hamlin'e 'Valência'. Para os dados ajustados de incidência da variedade 'Pera', o modelo monomolecular foi o único que não apresentou padrão no resíduo (Tabela 1). Devido a este resultado e por ter apresentado, na maioria das vezes, o coeficiente de determinação mais elevado, escolheu-se o modelo monomolecular para a comparação das variedades (Tabela 1). Além disso, este modelo tem sido recomendado para o ajuste de dados de doenças com período de incubação variável (Bergamin Filho \& Amorim, 2002), como é o caso da MPC, para a qual a incubação é mais dependente da fenologia do hospedeiro do que da época de infecção. 
TABELA 1 - Coeficientes de determinação $\left(R^{2}\right)$ e presença $(+)$ ou ausência (-) de padrão no resíduo após ajuste dos modelos monomolecular, logístico e Gompertz, aos dados de incidência e de severidade da mancha preta dos citros em laranja doce (Citrus sinensis) nas variedades 'Hamlin', 'Pera’ e 'Valência'

\begin{tabular}{llllllc}
\hline \hline \multirow{2}{*}{$\begin{array}{l}\text { Variedade de } \\
\text { laranja doce }\end{array}$} & \multirow{2}{*}{ Modelo } & \multicolumn{2}{c}{ Incidência } & & \multicolumn{2}{c}{ Severidade } \\
\cline { 3 - 4 } \cline { 6 - 7 } & & $\boldsymbol{R}^{2}$ & Resíduo & & $\boldsymbol{R}^{2}$ & Resíduo \\
\hline 'Hamlin' & monomolecular & 0,87 & - & & 0,71 & - \\
& logístico & 0,87 & - & & 0,71 & - \\
& Gompertz & 0,88 & & & 0,71 & \\
'Pera' & monomolecular & 0,93 & - & & 0,78 & - \\
& logístico & 0,89 & + & & 0,81 & - \\
& Gompertz & 0,91 & + & & 0,80 & - \\
'Valência' & monomolecular & 0,89 & - & & 0,77 & - \\
& logístico & 0,86 & - & & 0,77 & - \\
& Gompertz & 0,88 & - & & 0,77 & - \\
\hline
\end{tabular}

As curvas médias de progresso de incidência da MPC, produzidas pelo modelo monomolecular, foram bastante similares nas três variedades avaliadas (Figura 1A, C, E). O período de avaliação da variedade 'Hamlin' foi mais curto do que nas demais variedades em virtude do amadurecimento precoce e conseqüentemente da colheita de seus frutos. As curvas de progresso da severidade da MPC foram similares nas variedades 'Hamlin' e 'Valência' (Figura 1B, F), mas a variedade 'Pera' mostrou maior severidade final que as demais (Figura 1D). Entretanto, esta diferença não foi perceptível quando os parâmetros do modelo foram comparados pelo teste $t$. Os valores da assíntota máxima $\left(b_{1}\right)$ para as três variedades, obtidos para a severidade da doença, não diferiram entre si a $5 \%$ de probabilidade (Tabela 2). As constantes de integração $\left(b_{2}\right)$, relacionadas ao inóculo inicial da doença, obtidas para as curvas de progresso da incidência e severidade da MPC, foram significativamente maiores para a variedade 'Hamlin' (Tabela 2). A expressão dos sintomas da MPC está diretamente relacionada com o amadurecimento dos frutos (Feichtenberger, 1996). O maior valor do inóculo inicial na variedade 'Hamlin', representado por $b_{2}$, provavelmente deveu-se ao fato desta variedade iniciar o amadurecimento dos frutos antes das demais variedades. No início das avaliações, os frutos da variedade 'Hamlin' apresentaram um maior diâmetro e casca com cor verde menos intensa quando comparados com os das outras variedades (Figura 2). Esse resultado, entretanto, não exprime as diferenças na suscetibilidade dessas variedades à MPC.

Na epidemiologia comparativa, o parâmetro utilizado para diferenciar a suscetibilidade das variedades é a taxa de
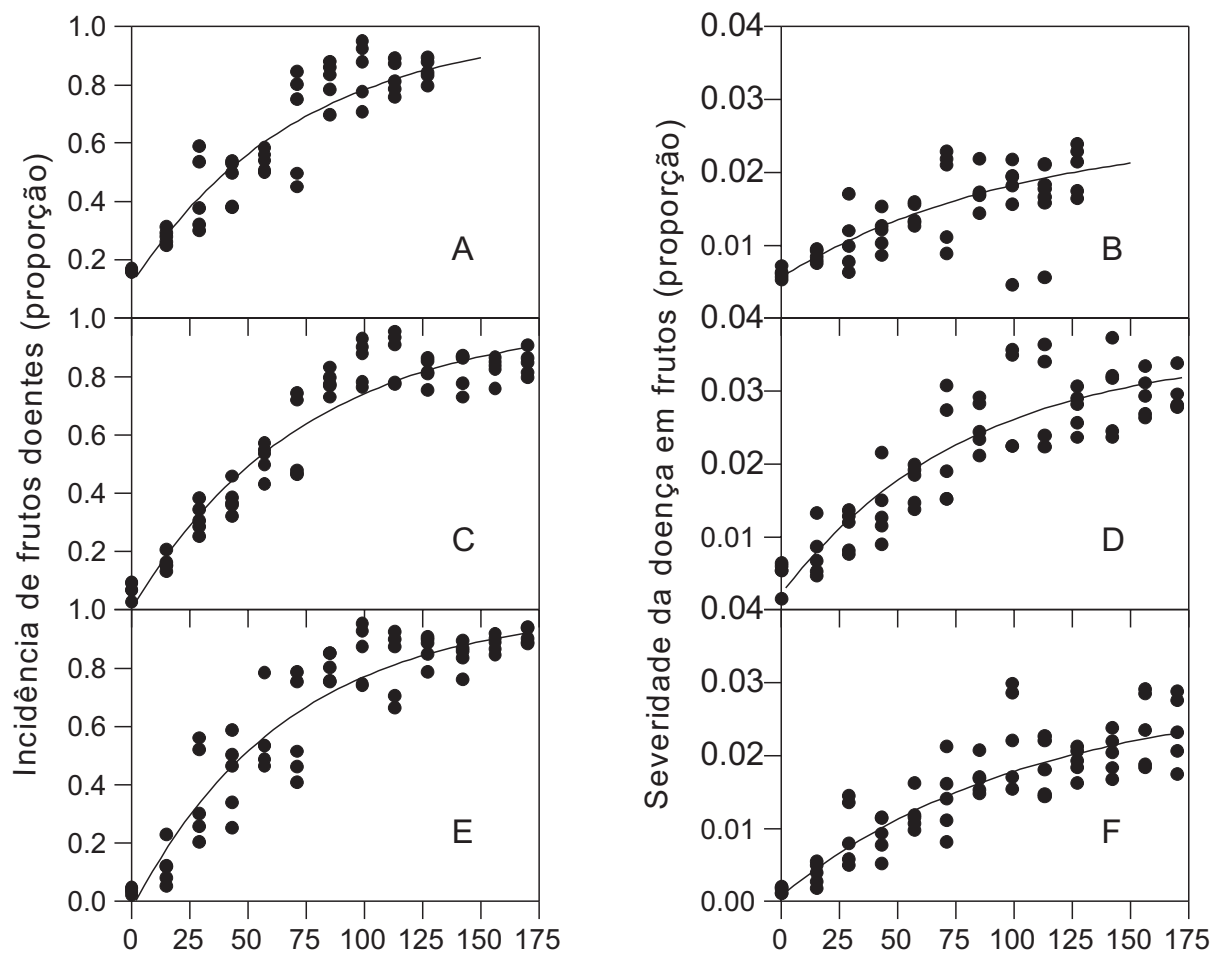

Tempo (dias após a primeira avaliação)

FIG. 1 - Curvas de progresso da incidência (A, C, E) e da severidade (B, D, F) de mancha preta dos citros em laranja doce (Citrus sinensis) nas variedades 'Hamlin' (A-B), 'Pera' (C-D) e 'Valência' (E-F) avaliadas a cada 15 dias a partir de 13 de março de 2001 até o período da respectiva colheita. Pontos representam dados médios de 20 plantas de cada repetição obtidos por avaliação e linhas representam o ajuste do modelo monomolecular. 
TABELA 2 - Assíntota máxima $\left(b_{1}\right)$, inóculo inicial $\left(b_{2}\right)$ e taxa de progresso da doença $(r)$ estimados pelo modelo monomolecular, para os dados de incidência e severidade nas variedades de laranjeiras doce (Citrus sinensis) 'Hamlin', 'Pera’ e 'Valência'

\begin{tabular}{|c|c|c|c|c|c|c|c|}
\hline \multirow{2}{*}{$\begin{array}{l}\text { Variedade de } \\
\text { laranja doce }\end{array}$} & \multicolumn{3}{|c|}{ Incidência * } & \multicolumn{4}{|c|}{ Severidade * } \\
\hline & $b_{1}$ & $b_{2}$ & $r$ & $b_{1}$ & $b_{2}$ & $R$ & \\
\hline 'Hamlin' & 1 & $0,13 \mathrm{a}$ & $0,014 \mathrm{a}$ & $0,026 \mathrm{a}$ & $0,22 \mathrm{a}$ & 0,0096 & $\mathrm{a}$ \\
\hline 'Pera' & 1 & $0,02 \mathrm{~b}$ & $0,013 \mathrm{a}$ & $0,038 \mathrm{a}$ & $0,08 \mathrm{~b}$ & 0,0107 & $\mathrm{a}$ \\
\hline 'Valência' & 1 & $0,00 \mathrm{~b}$ & $0,015 \mathrm{a}$ & $0,029 \mathrm{a}$ & $0,04 \quad \mathrm{c}$ & 0,0097 & $\mathrm{a}$ \\
\hline
\end{tabular}

* Dentro da coluna, valores com mesma letra diferem significativamente dos demais pelo teste $t$ a $5 \%$ de probabilidade.

progresso da doença. A taxa de progresso tanto da incidência quanto da severidade da MPC para as variedades 'Hamlin', 'Pera' e ‘Valência' não diferiram entre si a 5\% de probabilidade pelo teste $t$ (Tabela 2). Esse resultado indica que a doença cresceu na mesma velocidade nas três variedades e que, portanto, as três variedades apresentaram um mesmo grau de suscetibilidade para a mancha preta dos citros.

Os frutos da variedade 'Hamlin' alcançaram seu tamanho final e o amadurecimento externo (diâmetro médio dos frutos e índice de cor da casca, respectivamente) antes das variedades 'Pera' e 'Valência', as quais mostraram-se similares ao longo do tempo (Figura 2). Como o fungo G. citricarpa após infetar os frutos cítricos permanece na região subcuticular na forma de micélio quiescente (Feichtenberger, 1996) e o sintoma da doença restringe-se à região do flavedo (Cardoso Filho, 2003), correlacionou-se o índice de cor da casca com a severidade e com a incidência da mancha preta para as variedades de laranja doce 'Hamlin', 'Pera’ e 'Valência' (Figura 3). A correlação foi alta para as três variedades, entretanto o padrão observado para a 'Hamlin' diferiu das demais variedades (Tabela 3). Os sintomas da doença iniciaram-se com os frutos ainda verdes e intensificaram-se com a mudança da coloração da casca. Para as variedades 'Pera' ' 'Valência', o incremento da doença deuse até os frutos alcançarem um índice de cor entre -4 e -2 (mudança de cor do verde para o amarelo), estabilizando-se acima destes valores. Na variedade 'Hamlin' o incremento da doença foi quase linear desde os frutos totalmente verdes (-8), até a coloração laranja (+8), época de sua colheita (Figura 3). Nas variedades 'Pera' e 'Valência' a melhor época para a avaliação dos experimentos, na qual a incidência e a severidade de MPC nos frutos são máximas, é quando a cor da casca dos frutos atingem o valor zero. Neste caso a avaliação pode ser efetuada antes do período de colheita. Para a 'Hamlin' a incidência e a severidade máximas só são obtidas na época de colheita (incremento contínuo da doença com o incremento do índice de cor dos frutos) (Figura 3).

Os frutos cítricos possuem dois processos de desenvolvimento, resultando em sua maturação, um processo interno onde ocorre um aumento gradual de sólidos solúveis totais do

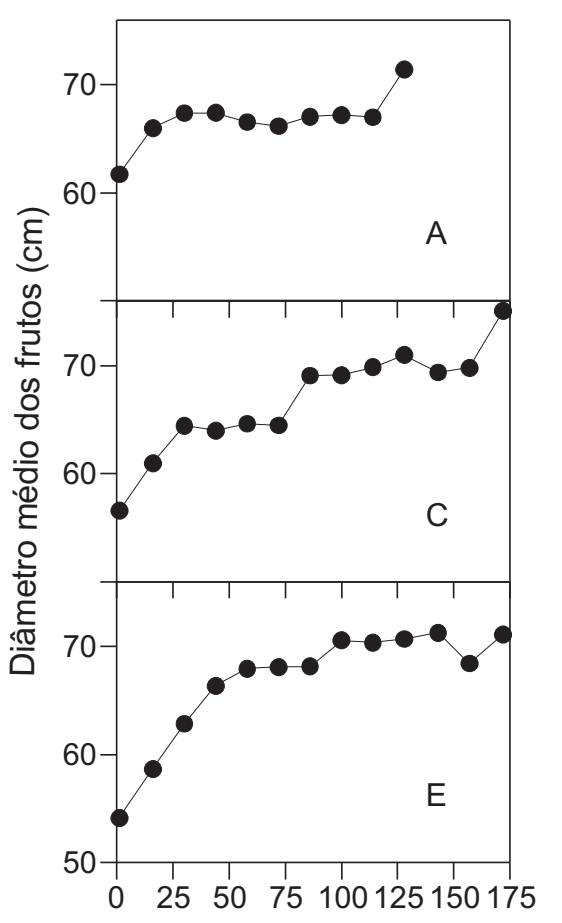

Tempo (dias após primeira avaliação)

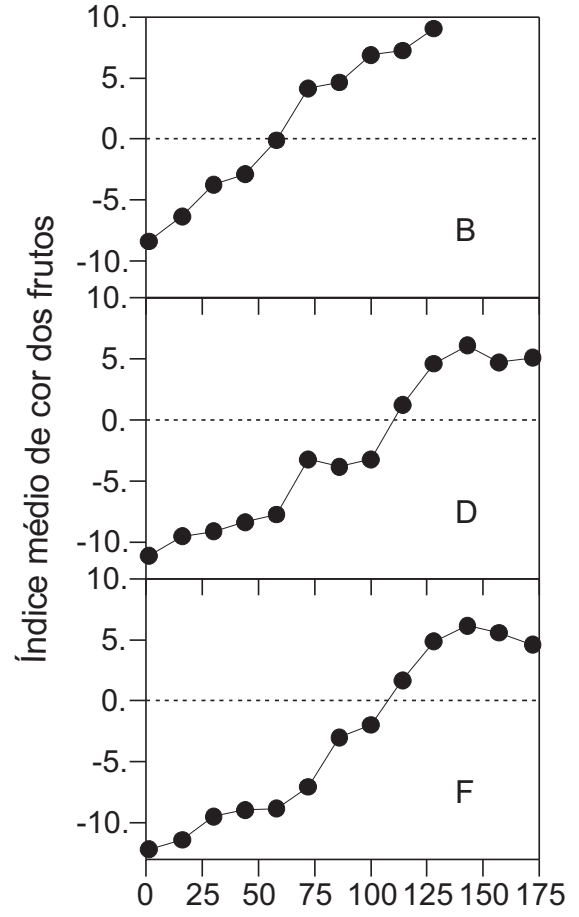

Tempo (dias após primeira avaliação)

FIG. 2 - Variação do diâmetro médio (A, C, E) e do índice médio de cor (B, D, F) dos frutos em laranja doce (Citrus sinensis) das variedades 'Hamlin' (A-B), 'Pera' (C-D) e 'Valência' (E-F) avaliados a cada 15 dias, a partir de 13 de maio de 2001 até o período da respectiva colheita. 
M.B. Spósito et al.

TABELA 3 - Coeficientes de determinação $\left(R^{2}\right)$ e parâmetros $a, b$ e $c$ das equações de segundo grau, $y=a x^{2}+b x+c$, onde $y$ representa a intensidade da doença (incidência e severidade) e $x$, o índice médio de cor da casca dos frutos em laranja doce (Citrus sinensis) variedades 'Hamlin', 'Pera’ e 'Valência'

\begin{tabular}{lccccccccc}
\hline Variedade de & \multicolumn{4}{c}{ Incidência } & & \multicolumn{4}{c}{ Severidade } \\
\cline { 2 - 5 } \cline { 7 - 10 } laranja doce & $\boldsymbol{a}$ & $\boldsymbol{b}$ & $\boldsymbol{c}$ & $\boldsymbol{R}^{\mathbf{2}}$ & $\boldsymbol{a}$ & $\boldsymbol{b}$ & $\boldsymbol{c}$ & $\boldsymbol{R}^{\mathbf{2}}$ \\
\hline 'Hamlin' & $-0,09$ & 4,10 & 57,79 & 0,98 & $-0,01$ & 0,08 & 1,41 & 0,99 \\
'Pera' & $-0,48$ & 1,82 & 86,14 & 0,93 & $-0,02$ & 0,06 & 3,04 & 0,93 \\
'Valência' & $-0,43$ & 1,54 & 90,06 & 0,98 & $-0,01$ & 0,05 & 2,09 & 0,94 \\
\hline
\end{tabular}

suco junto com uma diminuição de sua acidez total, e um processo externo em que ocorre a degradação da clorofila, por temperaturas altas, e a síntese de carotenos, por temperaturas baixas (Davies \& Albrigo, 1999). Esses dois processos são independentes e ocorrem conjuntamente, entretanto ambos são dependentes do ambiente e dos genótipos da copa e do porta-enxerto utilizados.

O mesmo grau de suscetibilidade à mancha preta dos citros entre as variedades 'Hamlin', 'Pera'e 'Valência', obtidos pela comparação das taxas de progresso da doença $(r)$, assim como as correlações encontradas entre o índice de cor e a severidade ou a incidência da doença obtidos neste experimento, exprimem a relação existente entre o processo de maturação dos frutos cítricos, dependente das condições ambientais, com a intensidade de expressão dos sintomas da doença.

\section{REFERÊNCIAS BIBLIOGRÁFICAS}

AGUILAR-VILDOSO, C.I., RIBEIRO, J.G.B., FEICHTENBERGER, E., GOES, A. \& SPÓSITO, M.B. Manual Técnico de Procedimentos da Mancha Preta dos Citros. Brasília. MAPA/SDA/DDIV. 2002.

ALCOBA, N.J., VIGIANI, A.R., BEJARANO, N.V., ALVAREZ, S.E., SERRANO, M.A. \& BONILLO, M.C. La Mancha Negra de los Citricos. Jujuy. Ed. Universidad Nacional de Jujuy. 2000.

BERGAMIN FILHO, A. \& AMORIM, L. Doenças de plantas tropicais: Epidemiologia e controle econômico. São Paulo. Ed. Ceres. 1996.

BERGAMIN FILHO, A. \& AMORIM, L. Doenças com período de incubação variável em função da fenologia do hospedeiro. Fitopatologia Brasileira 27:561-565. 2002.

CAMPBELL, C.L. \& MADDEN, L.V. Introduction to plant disease epidemiology. New York. Wiley-Interscience. 1990.

CARDOSO FILHO, J.A. Efeito de extratos de albedo de laranja (Citrus sinensis) e dos indutores de resistência ácido salicílico, acilbenzolar-s-metil e Saccharomyces cerevisiae no controle de Phyllosticta citricarpa (teleomorfo: Guignardia citricarpa). (Tese de Doutoramento). Piracicaba. Escola Superior de Agricultura "Luiz de Queiroz”, Universidade de São Paulo. 2003.

CORNELL, J.A. \& BERGER, R.D. Factors that influence the coefficient of determination in single linear and nonlinear models. Phytopathology 77:63-70.1987.

DAVIES, F.S. \& ALBRIGO, L.G. Cítricos. Zaragoza. Ed. Acribia. 1999. FEICHTENBERGER, E. Mancha-preta dos citros no Estado de São Paulo. Laranja 17:93-108.1996.

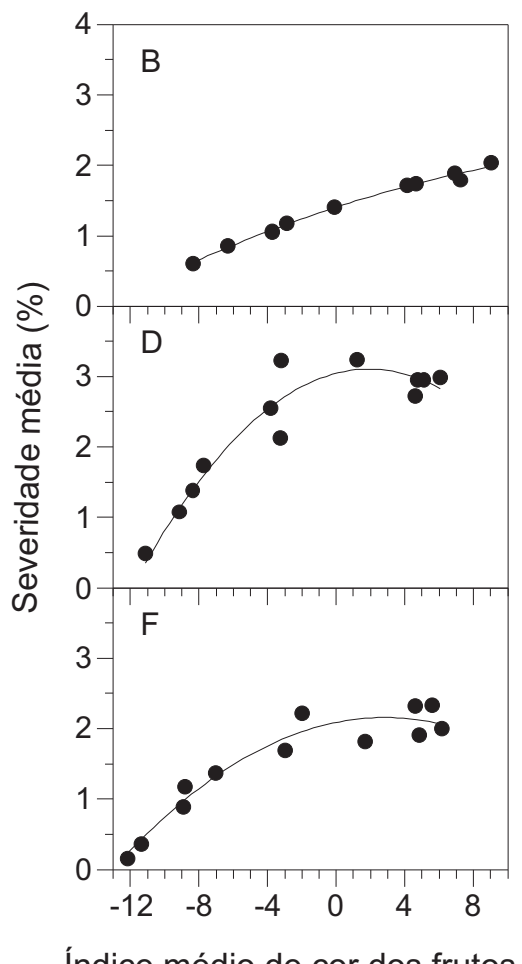

Índice médio de cor dos frutos

FIG. 3 - Variação da incidência e severidade de mancha preta em relação ao índice de cor em frutos em laranja doce (Citrus sinensis) variedades ‘Hamlin’ (A-B), ‘Pera’ (C-D) e ‘Valência’ (E-F). 
Resistência à mancha preta dos citros avaliada por curvas...

FNP Consultoria \& Comércio. Agrianual 2002. Anuário da Agricultura Brasileira. São Paulo. Ed. Argos. 2002.

GOES, A. \& FEICHTENBERGER, E. Ocorrência da mancha preta causada por Phyllosticta citricarpa (Guignardia citricarpa) em pomares cítricos do Estado de São Paulo. Fitopatologia Brasileira 15:73-75. 1993.

GOES, A. Controle da mancha-preta dos frutos cítricos. Laranja 19:305320. 1998.

KEINATH, A.P. \& FARNHAM, M.W. Differential cultivars and criteria for evaluating resistance to Rhizoctonia solani in seedling Brassica oleracea. Plant Disease 81:946-952. 1997.

KIELY, T.B. Preliminary studies on Guignardia citricarpa n. sp.: The ascigerous stage of Phoma citricarpa McAlp. and its relation to black spot of citrus. Proceedings of Linnean Society of New South Wales 73:249-292. 1948.

KLOTZ, L.J. Fungal, bacterial and nonparasitic diseases and injuries originating in the seedbed, nursery and orchard. In: Reuther, W., Calavan, E.C. \& Carman, G.E. (Eds.) The Citrus Industry v. 4. Davis. University of California. 1978. pp.1-66.

KOTZÉ, J.M. Studies on the black spot disease of citrus caused by Guignardia citricarpa Kiely with particular reference to its epiphytology and control at Lebata. (PhD Thesis). Pretoria. University of Pretoria. 1963.

KRANZ, J. Comparasion of epidemics. Annual Review of Phytopathology 12:355-374. 1974.

MAZZUZ, C.F. Calidad de Frutos Citricos. Manual para su Gestión desde la Recolección hasta la Expedición. Reus. Ed. Horticultura. 1996.

NETER, J., KUTNER, M.H., NACHTSHEIM, C.J. \& WASSERMAN, W. Applied Linear Statistical Models. $4^{\text {th }}$ ed. Chicago. Irwin. 1996.

SILVA, M.B., VALE, F.X.R., ZAMBOLIM, L. \& PAULA JR., T.J. Caracterização da curva de progresso da ferrugem do feijão em diferentes épocas de plantio em Viçosa, MG. Fitopatologia Brasileira 23:139-142. 1998.

SPÓSITO, M.B., AMORIM, L., BELASQUE JR., J., BASSANEZI, R. B. \& AQUINO, R. Elaboração e validação de escala diagramática para avaliação da severidade da mancha preta em frutos cítricos. Fitopatologia Brasileira 28:S231. 2003. (Resumo)

TIMMER, L. W. Diseases of fruit and foliage. In: Timmer, L.W. \& Duncan, L.W. (Eds.) Citrus Health Management. Saint Paul. APS Press. 1999. pp.107-115. 